\title{
Spirochete-platelet attachment and thrombocytopenia in murine relapsing fever borreliosis
}

Kishore R. Alugupalli, Alan D. Michelson, Isabelle Joris, Tom G. Schwan, Kairbaan Hodivala-Dilke, Richard O. Hynes, and John M. Leong

Thrombocytopenia is common in persons infected with relapsing fever Borreliae. We previously showed that the relapsing fever spirochete Borrelia hermsii binds to and activates human platelets in vitro and that, after platelet activation, high-level spirochete-platelet attachment is mediated by integrin $\alpha_{\| b b} \beta_{3}$, a receptor that requires platelet activation for full function. Here we established that $B$ hermsii infection of the mouse results in severe thrombocytopenia and a functional defect in hemostasis caused by accelerated platelet loss. Disseminated intravas- cular coagulation, immune thrombocytopenic purpura, or splenic sequestration did not play a discernible role in this model. Instead, spirochete-platelet complexes were detected in the blood of infected mice, suggesting that platelet attachment by bacteria might result in platelet clearance. Consistent with this, splenomegaly and thrombocytopenia temporally correlated with spirochetemia, and the severity of thrombocytopenia directly correlated with the degree of spirochetemia. Activation of platelets and integrin $\alpha_{\| l b} \beta_{3}$ were apparently not required for bacterium-platelet binding or platelet clearance because the bacterium-bound platelets in the circulation were not activated, and platelet binding and thrombocytopenia during infection of $\boldsymbol{\beta}_{3}$-deficient and wild-type mice were indistinguishable. These findings suggest that thrombocytopenia of relapsing fever is the result of platelet clearance after $\boldsymbol{\beta}_{3}$-independent bacterial attachment to circulating platelets. (Blood. 2003;102:2843-2850)

๑) 2003 by The American Society of Hematology

\section{Introduction}

Thrombocytopenia can be a complication of many viral, bacterial, fungal, and protozoan infections. ${ }^{1}$ Platelets, produced in the bone marrow by megakaryocytes, are central to primary hemostasis because of their role in maintaining endothelial integrity. ${ }^{2}$ In some instances, infection-induced thrombocytopenia is severe enough to cause bleeding. Thrombocytopenia associated with bacterial infections is often thought to occur secondarily to disseminated intravascular coagulation (DIC), a generalized activation of the coagulation cascade during overwhelming bacteremia that results in the rapid consumption of platelets. ${ }^{1}$ Thrombocytopenia associated with viral infections is often caused by the generation of antiplatelet antibodies, with subsequent accelerated platelet clearance, or by the suppression of megakaryopoiesis. ${ }^{3,4}$ However, a significant percentage of patients with infection-induced thrombocytopenia have no evidence of DIC, antiplatelet antibodies, or bone marrow suppression. ${ }^{1,5-7}$ One proposed model for thrombocytopenia during infections involves the formation of platelet-microbe complexes, followed by the clearance of these complexes from the circulation. ${ }^{8-12}$

To investigate how high-level growth of microbes in the blood might lead to thrombocytopenia, we studied relapsing fever caused by arthropod-borne spirochetes of the genus Borrelia. ${ }^{13}$ Although these spirochetes can infect a number of organs, such as the spleen, liver, heart, and brain, the hallmark of relapsing fever is recurrent high-level (eg, 108/mL) spirochetemia. Each episode of spirochetemia is caused by a serologically distinct population of bacteria. Serotype variation results from the expression of different variable major surface proteins, and it involves gene replacement at a specific expression site. ${ }^{14}$ The progeny of a single bacterium encode many silent variable major protein genes, and as many as 26 antigenically distinct serotypes of this bacterium can be generated in a single infection. ${ }^{15}$

Interestingly, thrombocytopenia is a prominent feature of relapsing fever. Petechial rash, epistaxis, hematuria, and mucosal bleeding are common findings in louse-borne relapsing fever. ${ }^{16-19}$ In North America, relapsing fever is caused mainly by tick-borne spirochetes such as Borrelia hermsii and Borrelia turicatae, and thrombocytopenia is the most common laboratory finding in infected patients. ${ }^{20}$

Given that relapsing fever spirochetes cause thrombocytopenia and, by growing to high concentrations in the blood, have ample opportunity to interact with blood cells, we have been investigating the interaction of $B$ hermsii with platelets. ${ }^{12}$ Circulating platelets are found in a relatively nonadhesive "resting" state. Activation of platelets by thrombin, adenosine diphosphate (ADP), or other agonists dramatically increases platelet adhesiveness, ${ }^{21}$ in part by altering the conformation of the major platelet integrin $\alpha_{\mathrm{IIb}} \beta_{3}$ to a
From the Department of Molecular Genetics and Microbiology, Center for Platelet Function Studies/Department of Pediatrics, and Department of Pathology, University of Massachusetts Medical School, Worcester, MA; Laboratory of Human Bacterial Pathogenesis, Rocky Mountain Laboratories, National Institute of Allergy and Infectious Diseases, Hamilton, MT; and Center for Cancer Research and Department of Biology, Massachusetts Institute of Technology, Cambridge, MA.

Submitted February 7, 2003; accepted June 26, 2003. Prepublished online as Blood First Edition Paper, July 10, 2003; DOI 10.1182/blood-2003-02-0426.

Supported by National Institutes of Health grants R01-AI 37601 (J.M.L.), and P01HL66105 (R.O.H.), and supported in part by Diabetes and Endocrinology
Research Center grant DK 32520 (I.J.). J.M.L. was an Established Investigator of the American Heart Association. R.O.H. is an investigator for the Howard Hughes Medical Institute.

Reprints: John M. Leong, Department of Molecular Genetics and Microbiology, University of Massachusetts Medical School, 55 Lake Ave N, Worcester, MA 01655; e-mail: john.leong@umassmed.edu.

The publication costs of this article were defrayed in part by page charge payment. Therefore, and solely to indicate this fact, this article is hereby marked "advertisement" in accordance with 18 U.S.C. section 1734

(C) 2003 by The American Society of Hematology 
high-affinity state. ${ }^{22}$ We previously found that $B$ hermsii was capable of contact-dependent platelet activation and that, after platelets had been activated, the spirochetes bound efficiently through integrin $\alpha_{\mathrm{IIb}} \beta_{3} .{ }^{12}$

The murine model of relapsing fever borreliosis recapitulates a number of pathophysiologic aspects of the human disease and has been used to investigate pathways of immune clearance by the host $^{23-25}$ and mechanisms of immune evasion ${ }^{14,15}$ and tissue invasion by the microbe. ${ }^{26}$ Recent studies indicate that thrombocytopenia also develops in experimentally infected mice. ${ }^{26-28}$ In the current study, we have investigated thrombocytopenia in the murine model of relapsing fever and have found no evidence of bone marrow suppression, systemic activation of coagulation, or antiplatelet antibodies. Instead, $B$ hermsii-induced thrombocytopenia may result from a direct interaction between bacteria and circulating platelets.

\section{Materials and methods}

\section{Borrelia strains}

$B$ hermsii DAH was isolated from a patient with relapsing fever northwest of Cheney, WA. ${ }^{29}$ Notably, this patient had thrombocytopenia (platelet count, $89000 / \mu \mathrm{L}$ blood) during her acute illness. After treatment and recovery from infection, the patient's platelet count returned to within normal limits $(410000 / \mu \mathrm{L}$ blood). $B$ hermsii HS1, a tick isolate from Spokane, WA, ${ }^{30}$ was shown previously to efficiently bind and activate human platelets. ${ }^{12}$ Strains DAH and HS1 are indistinguishable by restriction fragment length polymorphism analysis. ${ }^{31} \mathrm{~B}$ hermsii strains FRO, HAN, MAN, and CON are clinical isolates from the western United States and were previously described. ${ }^{31} B$ hermsii HS1-Vlp7 was obtained from Dr Alan Barbour, University of California, Irvine.

\section{Mice}

The 129Sv/C57BL6 control mice and $\beta_{3}$-deficient mice ${ }^{32}$ were bred and maintained in micro-isolator cages in the Department of Animal Medicine at the University of Massachusetts Medical School (UMMS). Normal 4-week-old C57BL6 and C57BL6/SCID mice and mice of the same age that underwent splenectomy were purchased from Jackson Laboratories (Bar Harbor, ME), whereas CB.17/SCID mice were provided by Dr Robert Woodland (UMMS).

\section{Murine infections}

Three- to 5-week-old mice were inoculated intraperitoneally with $10^{6}$ spirochetes in $100 \mu \mathrm{L}$ BSK-H medium (Sigma, St Louis, MO), unless stated otherwise. Control mice were injected with an equal volume of sterile BSK-H medium. To monitor spirochetemia and thrombocytopenia, an aliquot of $5 \mu \mathrm{L}$ blood was sampled from the tail bleed and diluted immediately into $45 \mu \mathrm{L}$ citrated anticoagulant $(0.11 \mathrm{M}$ sodium citrate/citric acid in phosphate-buffered saline [PBS]). ${ }^{12,28}$ A portion of the sample was examined under dark-field microscope (magnification, $\times 400$ ) to quantify spirochetemia. $^{26}$

\section{Quantitation of platelets and platelet-bacterium complexes and characterization of platelet activation}

Flow cytometry was used to enumerate platelets ${ }^{28}$ and to identify and quantify bacterium-platelet complexes in the circulations of infected mice. To identify bacteria and platelets, $10 \mu \mathrm{L}$ anticoagulated blood sample described above was stained with phycoerythrin (PE)-labeled antimouse platelet CD61 mAb ( $\beta_{3}$-integrin) (clone 2C9.G2; PharMingen, San Diego, $\mathrm{CA}$ ) or CD49b mAb (clone $\mathrm{Hm} \alpha 2$; PharMingen) and fluorescein isothiocyanate (FITC)-conjugated $B$ hermsii antibodies. ${ }^{12}$ Samples were fixed in $0.1 \%$ formalin in PBS, and platelets with bound bacteria were identified by their FITC fluorescence above the threshold value, set by using an identically stained sample of uninfected murine blood. We previously showed that staining of platelet-bacterium complexes formed in vitro gives rise to a continuum of fluorescence, reflecting both a continuum of bacterial binding as assessed microscopically ${ }^{12}$ and a continuum of immunoreactivity. In vitro-cultivated $B$ hermsii stained variably with the antiserum used in this study, as assessed by flow cytometry, whereas bacteria harvested from infected mice demonstrated uniform immunoreactivity (data not shown). The difference in immunoreactivities of these populations of bacteria may be that the major surface antigen (the variable major protein) expressed by bacteria cultivated in vitro is distinct from that expressed by host-adapted bacteria. ${ }^{29}$

To assess platelet activation, blood samples were stained with biotinylated antimouse CD62P (P-selectin, clone RB40.34; PharMingen) and PE-labeled antimouse platelet CD61 mAb ( $\beta_{3}$-integrin) followed by streptavidin-labeled RED670 (Gibco BRL, Bethesda, MD). As positive control for platelet activation, $10 \mu \mathrm{L}$ anticoagulated blood described above was treated without or with platelet agonist phorbol myristic acetate (PMA; $20 \mu \mathrm{M}) .^{28}$ At least 20000 platelet events were analyzed using a FACSCalibur flow cytometer (Becton Dickinson, Mountain View, CA) equipped with a 15-mW, 488-nm, air-cooled argon laser, standard 3-color filter arrangement, and CELLQuest software (Becton Dickinson). Positive and negative controls were used to establish color compensation settings using appropriately tagged, isotype-matched, nonspecific antibodies. Bacterial binding did not diminish the staining (mean fluorescence intensity) of activated platelets by anti-P-selectin or anti-integrin $\alpha_{\text {IIb }}$ (the subunit of integrin $\left.\alpha_{I I b} \beta_{3}\right)^{12}$ (and data not shown).

To assess the response of the residual platelets of thrombocytopenic mice, platelets from these mice and uninfected mice were purified as described ${ }^{33}$ Both platelet preparations were incubated with ADP $(40 \mu \mathrm{M}$; Bio/Data, Horsham, PA), epinephrine (5 $\mu \mathrm{M}$; Bio/Data) and collagen (20 $\mu \mathrm{g} / \mathrm{mL}$; Chrono-log, Havertown, PA), or thrombin (0.2 U/mL; Chrono-log) for 30 minutes at room temperature, and $\mathrm{P}$-selectin expression or $\beta_{3}$ integrin up-regulation was measured.

\section{Analysis of antibody-mediated induction of thrombocytopenia}

To examine a possible involvement of antiplatelet antibody in the induction of thrombocytopenia, serum was harvested from wild-type mice 3 days after infection, when they had severe thrombocytopenia (platelet levels approximately $10 \%$ of normal). The serum was filter-sterilized, and $300 \mu \mathrm{L}$ was injected intravenously into naive mice. Platelet counts were monitored by flow cytometry. ${ }^{28}$

\section{Determination of accelerated clearance of platelets}

Mouse platelets were biotinylated in vivo by intravenous infusion of sulfo- $N$-hydroxysuccinimido biotin (Pierce, Rockford, IL) as described. ${ }^{33,34}$ After 2 hours, the infused mice were infected with $B$ hermsii as described above. An aliquot of $5 \mu \mathrm{L}$ blood was sampled at various times, diluted, stained with PE-labeled antimouse platelet CD61 mAb and FITC-labeled streptavidin, and analyzed by flow cytometry to determine the numbers of biotinylated and nonbiotinylated platelets.

\section{Attachment of $B$ hermsii to murine platelets in vitro}

Platelets from wild-type or $\beta 3$-deficient mice were isolated as described earlier. ${ }^{33}$ Activated platelets were prepared by adding $0.1 \mathrm{U}$ thrombin to 500 $\mu \mathrm{L}$ platelets, followed by incubation for 20 minutes at room temperature. Bacteria were incubated with platelets at a multiplicity of infection of approximately 2 in Tyrode buffer containing $0.2 \%$ bovine serum albumin (BSA) for 60 minutes at room temperature. ${ }^{12}$ Where indicated, EDTA (ethylenediaminetetraacetic acid; final concentration, $10 \mathrm{mM}$ ) was added to platelets for 15 minutes before binding. Bacteria-bound platelets were quantitated by flow cytometry as described above.

\section{Bleeding-time measurements}

Bleeding time was determined as described by Dejana et al. ${ }^{35}$ In brief, $2 \mathrm{~mm}$ of the distal end of the tail was transected using a razor blade, and the tail 
was immersed in $0.9 \%$ saline at $37^{\circ} \mathrm{C}$. The time it took for the blood to cease streaming was recorded as the bleeding time.

\section{Hepatosplenomegaly and bone marrow analysis}

To determine the hepatosplenomegaly associated with $B$ hermsii infection, 16 mice were inoculated intraperitoneally with $10^{6}$ spirochetes. Two mice each were killed on days $0,2,3,4,6,8,10$, and 12 after infection, and their spleens and livers were excised and immediately weighed.

To count the megakaryocytes in the marrow, femurs were removed and fixed in $10 \%$ buffered formalin. Five-micrometer sagittal sections were cut and stained with hematoxylin and eosin. Morphologically identifiable megakaryocytes were counted by light microscopy in 20 fields (magnification, $\times 400) .{ }^{36}$

\section{Detection of fibrin D-dimers}

To detect fibrin D-dimers in the blood of $B$ hermsii-infected mice, groups of C57BL6 mice (5 mice/group) were injected intraperitoneally with saline (negative control) or lipopolysaccharide (LPS) (positive control; $500 \mu \mathrm{g}$ Escherichia coli O55:B5 LPS; Calbiochem, San Diego, CA) or B hermsii strain DAH ( $10^{6}$ spirochetes). After 48 hours, mice were anesthetized and blood was drawn by cardiac puncture into citrated anticoagulant. Plasma was isolated after centrifugation at $13000 \mathrm{~g}$ and was analyzed for the presence of fibrin D-dimers using enzyme-linked immunosorbent assay (ELISA) (Asserchrom D-Di; Diagnostica Stago, Asnieres, France). ${ }^{37}$

\section{Results}

\section{$B$ hermsii infection results in thrombocytopenia and prolonged bleeding}

To test whether $B$ hermsii causes thrombocytopenia in infected mice, 129Sv/C57BL6 mice were inoculated intraperitoneally with $10^{6}$ spirochetes, and platelet counts were determined during peak spirochetemia (day 2 after infection). Platelet counts in mockinfected mice were within the normal range for mice, ${ }^{28,38}$ whereas platelet counts were 5- to 20 -fold lower in infected mice (Table 1). To determine whether the decrease in platelet counts was associated with a functional defect in hemostasis, we measured the bleeding time in infected mice. The bleeding time in mock-infected mice was approximately 1 minute (Table 1 ). In contrast, infected mice demonstrated bleeding times at least 5 times longer, consistent with manifestations of bleeding observed in patients with relapsing fever. ${ }^{17-19}$ The responsiveness of the remaining platelets

Table 1. B hermsii infection results in thrombocytopenia and prolonged bleeding time in mice

Mouse no. Platelets $/ \mu \mathrm{L}, \times 10^{3}$ Bacteria/ $\mu \mathrm{L}, \times 10^{3}$ Bleeding time, $\mathrm{s}$

\begin{tabular}{crrr}
\hline Mock-infected & & & \\
\hline 1 & 810 & NA & 58 \\
2 & 900 & NA & 56 \\
3 & 1150 & NA & 52 \\
\hline 4 & 1100 & NA & 72 \\
\hline 5 & 1130 & NA & 60 \\
B hermsii-infected & & & \\
6 & 70 & 40.0 & $>600$ \\
7 & 60 & 26.6 & $>600$ \\
\hline 8 & 120 & 13.3 & 316 \\
\hline 9 & 210 & 7.0 & 292 \\
10 & 50 & 13.3 & $>600$ \\
\hline
\end{tabular}

Platelet and spirochete counts in blood were determined by flow cytometry and by visual counting, respectively. Bleeding times were measured on mock- or 2-day postinfected mice, as described in "Materials and methods." NA indicates no applicable. of the infected mice to the platelet agonists thrombin, collagen/ epinephrine, and ADP was comparable with that of the platelets of uninfected mice (data not shown), suggesting that the observed increase in bleeding time resulted from a quantitative rather than a qualitative platelet defect.

\section{Accelerated clearance of platelets in B hermsii-infected mice}

The decreased number of circulating platelets during relapsing fever indicates either decreased platelet production or increased platelet clearance. ${ }^{26,27,39}$ Diminished production of platelets from the suppression of megakaryopoiesis in the bone marrow can occur during infection ${ }^{40}$; therefore, we measured megakaryocyte numbers in the bone marrow of $B$ hermsii-infected mice. No decrease in the number of megakaryocytes was detected during thrombocytopenia. In fact, the megakaryocyte count increased more than 2 -fold during the initial period of thrombocytopenia, then returned to normal levels (Figure 1A). A rapid increase in megakaryocyte count in response to increased platelet clearance has previously been observed during experimental consumptive thrombocytopenia. ${ }^{36}$ These results indicate that $B$ hermsii-induced thrombocytopenia is not the result of bone marrow suppression, and by exclusion they suggest that thrombocytopenia is likely caused by accelerated platelet consumption.

To directly measure the clearance of platelets in $B$ hermsiiinfected mice, platelets (and other blood cells) were labeled in vivo by intravenous infusion of a biotinylation agent (see "Materials and methods"). On postinfection days 1 through 4, blood samples from $B$ hermsii-infected or uninfected mice were analyzed by flow cytometry to determine the number of biotinylated platelets (present in the circulation at the time of platelet labeling) and the number of nonbiotinylated platelets (newly synthesized) (Figure 1B). The steady rise of nonbiotinylated platelets and the steady fall of biotinylated platelets in uninfected mice is a reflection of normal platelet turnover. ${ }^{33,34}$ Examination of infected mice showed that by day 2 after infection, the clearance of biotinylated platelets was significantly faster in infected mice than in control mice. Similarly, the clearance of nonbiotinylated platelets was faster in infected than in control mice after postinfection day 2 . We conclude that the thrombocytopenia of relapsing fever is caused by accelerated platelet clearance and that the rate of clearance in infected mice is not dramatically influenced by platelet age.

\section{Thrombocytopenia induced by $B$ hermsii infection is not associated with generalized activation of the coagulation cascade}

Many, but not all, severe bacterial infections induce thrombocytopenia as a result of DIC, a condition precipitated by the generalized activation of the coagulation cascade in response to bacterial components such as LPS and peptidoglycan. ${ }^{1,5}$ This systemic activation of coagulation results in generalized thrombus formation and in the rapid consumption of platelets. During this process, the fibrin formed is digested by plasmin, resulting in the release of fibrin D-dimer fragments into the blood and providing a sensitive and specific marker for this condition. ${ }^{37,41}$ To determine whether the thrombocytopenia of relapsing fever is caused by DIC, fibrin D-dimers were measured in the plasma of C57BL6 mice infected with $B$ hermsii strain DAH. The D-dimer levels were not elevated compared with those of the background levels detected in salineinjected control mice (Figure 2). In contrast, when DIC was induced in control mice by LPS injection, fibrin D-dimers were elevated 6-fold over background levels. These results suggest that 
A

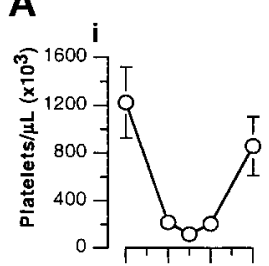

B
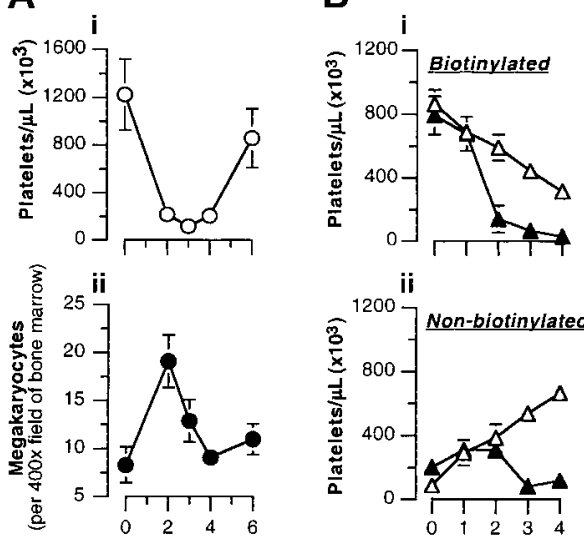

Days after infection

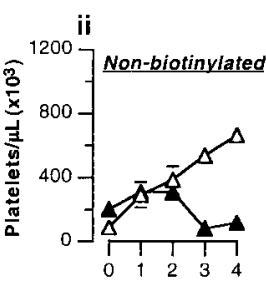

Days after infection

Figure 1. $B$ hermsii infection results in an increased rate of platelet clearance. (A) $B$ hermsii infection is not associated with diminished numbers of megakaryocytes. 129Sv/C57BL6 mice were inoculated intraperitoneally with $10^{6} B$ hermsii DAH. (i) On the indicated days after infection, platelet counts were determined by flow cytometry, and the mice were killed. (ii) Megakaryocytes in hematoxylin-eosinstained bone marrow sections were visually counted using light microscopy (original magnification, $\times 400$ ). Shown are the means \pm SDs from 20 fields. (B) Accelerated clearance of platelets in $B$ hermsii-infected mice. Numbers of biotinylated (i) and nonbiotinylated (ii) platelets in uninfected $(\triangle)$ or $B$ hermsii-infected $(\boldsymbol{\Lambda})$ mice at the indicated day after infection were determined by flow cytometry. Each curve represents the mean $\pm \mathrm{SD}$ of 5 mice.

the thrombocytopenia of relapsing fever is not a consequence of DIC.

\section{$B$ hermsii-induced thrombocytopenia does not require specific immunity}

Immune thrombocytopenic purpura, involving the generation of autoreactive platelet antibodies that lead to platelet-clearance, is another well-characterized pathway for the induction of thrombocytopenia during infection. ${ }^{42-44}$ To test whether antibody production was required for thrombocytopenia associated with relapsing fever, CB.17/SCID or C57BL6/SCID mice were infected intraperitoneally with $B$ hermsii strain DAH, and bacterial and platelet counts were monitored for 10 days. Infected mice became severely bacteremic by day 3 after infection, and all became severely thrombocytopenic (Figure 3A). To test whether any serum factors (eg, antibodies) were involved in the induction of thrombocytopenia, we harvested serum from wild-type mice on day 3 after infection, when they had severe thrombocytopenia. After filter sterilization, the serum was transferred intravenously to naive mice. No drop in platelet count was observed (Figure 3B). We
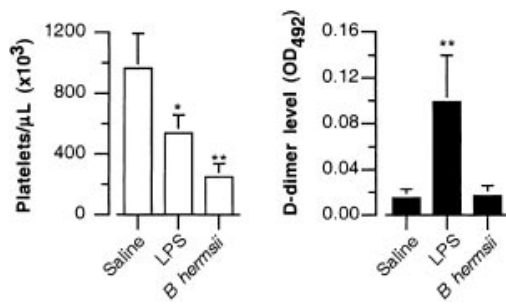

Figure 2. Thrombocytopenia induced by $B$ hermsii infection is not associated with generalized activation of the coagulation cascade. C57BL6 mice were injected intraperitoneally with control buffer, LPS, or $10^{6} \mathrm{~B}$ hermsii DAH. Two days after infection, platelet counts were determined by flow cytometry (left), and plasma fibrin D-dimer levels (right) were quantitated by ELISA (see "Materials and methods"). Shown are the means \pm SDs of 5 mice. ${ }^{*} P<.05$ compared with saline; ${ }^{* \star} P<.001$ compared with saline.
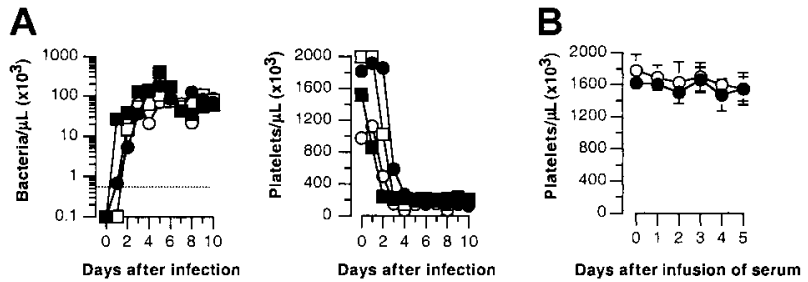

Figure 3. $B$ hermsii-induced thrombocytopenia does not require specific immunity. (A) Two CB.17/SCID $(\bullet, \square)$ or 2 C57BL6/SCID $(\bigcirc, \square)$ mice were infected intraperitoneally with $10^{6} \mathrm{~B}$ hermsii strain DAH. Bacteremia (left) was measured by visually counting the number of spirochetes in infected blood under dark-field microscopy, and platelet counts (right) were determined by flow cytometry (see "Materials and methods"). Broken line indicates the detection limit for spirochetemia. (B) Platelet counts of mice infused with serum of uninfected $(\bigcirc)$ and infected thrombocytopenic $(\bullet)$ mice. Shown are the means \pm SDs of 5 mice.

conclude that $B$ hermsii infection does not trigger thrombocytopenia by eliciting autoreactive platelet antibodies.

\section{$B$ hermsii binds to platelets during infection}

Interaction between microbes and platelets has been proposed as a mechanism for accelerated platelet clearance, ${ }^{8,45}$ and we recently demonstrated that $B$ hermsii binds to human platelets in vitro. ${ }^{12}$ To determine whether the bacterium-platelet binding that we have detected in vitro occurs in the blood of infected mice, we stained and fixed $B$ hermsii and platelets immediately after sampling blood from spirochetemic mice. Flow cytometric analysis of infected blood revealed that a significant percentage of platelets were found attached to spirochetes during infection (Figure 4). The actual percentage of bacterium-bound platelets varied from mouse to mouse but reached as high as $10 \%$ (data not shown). Microscopic examination of infected blood also revealed that bacterium-platelet complexes consisted entirely of one bacterium per platelet (data not shown).

\section{Thrombocytopenia is temporally correlated with spirochetemia}

If thrombocytopenia is a result of bacterium-platelet interaction, episodes of spirochetemia are predicted to temporally correlate with episodes of thrombocytopenia. To manipulate the kinetics of spirochetemia, we infected 3- to 5-week-old 129Sv/C57BL6 mice with decreasing numbers of $B$ hermsii strain DAH. Smaller inocula resulted in peak spirochetemia at later times: peak spirochetemia occurred at day 5 after infection with $10^{2}$ spirochetes (Figure 5Av) but at postinfection day 2 after inoculation with $10^{6}$ spirochetes (Figure 5Ai). In all cases, the course of thrombocytopenia followed

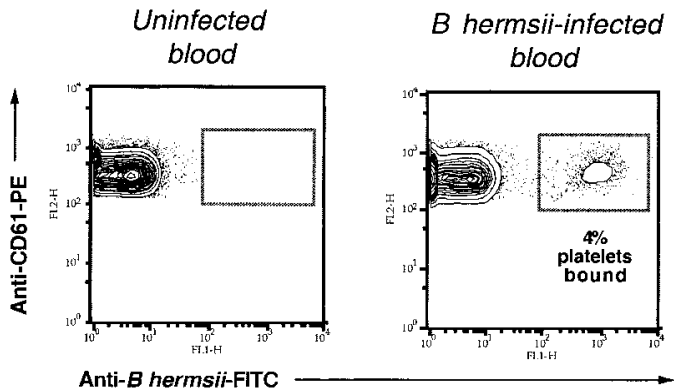

Figure 4. $B$ hermsii binds to platelets during infection. Blood from uninfected mice or mice infected with $10^{6} B$ hermsii DAH was analyzed at postinfection day 2 , that is, at peak spirochetemia. Samples were double-stained with FITC-conjugated anti-B hermsii antibody and PE-conjugated antiplatelet antibody. The percentage of platelets bound to $B$ hermsii was measured by flow cytometry, as described in "Materials and methods." 
Figure 5. Thrombocytopenia is temporally correlated with spirochetemia. (A) 129Sv/C57BL6 mice were inoculated intraperitoneally with decreasing numbers of $B$ hermsii DAH, as indicated. Spirochetemia (i-v) was measured by visually counting the number of spirochetes in the infected blood under dark-field microscopy, whereas platelet counts (vi-x) were determined by flow cytometry. The broken line indicates the detection limit for spirochetemia. (B) Mice inoculated intraperitoneally with $10^{6} \mathrm{~B}$ hermsii DAH, spirochetemia, and thrombocytopenia were monitored as described. ${ }^{28}$ Peaks of spirochetemia (labeled S1, S2, S3, and S4; top panel) were temporally associated with episodes of thrombocytopenia (labeled T1, T2, T3, and T4; bottom panel).
A

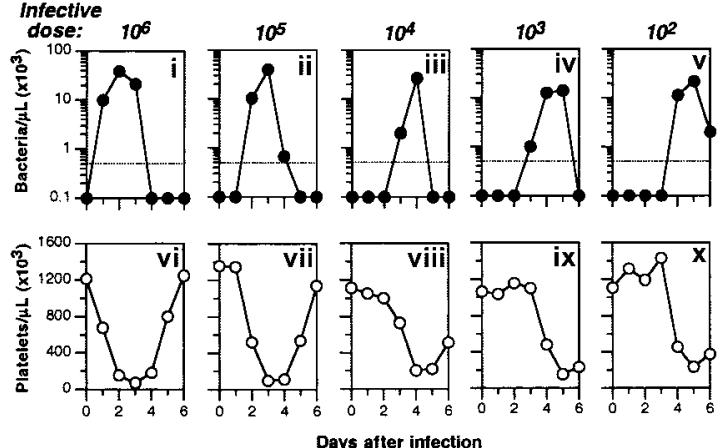

B
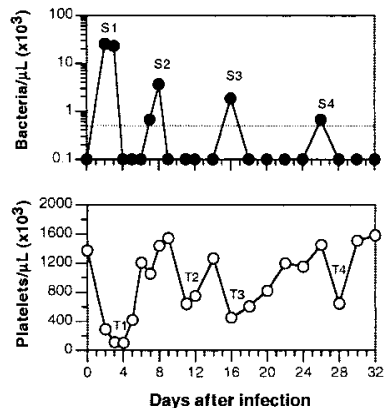

the course of spirochetemia-most severe on the day of or on the day after peak spirochetemia, and then diminishing in severity as spirochetemia diminished (Figure 5A).

The ability of $B$ hermsii to cause multiple episodes of spirochetemia because of its capacity for antigenic variation ${ }^{14,15}$ provided another opportunity to correlate spirochetemia and thrombocytopenia. 129Sv/C57BL6 mice inoculated with $10^{6}$ spirochetes had multiple spirochetemic peaks over a 4-week interval (Figure 5B). Each spirochetemic peak (S1-S4) in Figure 5B was closely followed by an episode of thrombocytopenia (T1-T4), demonstrating that thrombocytopenia and spirochetemia were temporally associated.

\section{Thrombocytopenia is quantitatively correlated with spirochetemia}

We previously showed that the degree of spirochetemia can vary with $B$ hermsii strain: low-passage $B$ hermsii strain DAH (designated DAH p1) grew to 50-fold higher concentrations in the blood than the same strain after 19 in vitro passages (DAH p19). ${ }^{12}$ To determine whether thrombocytopenia is quantitatively correlated with spirochetemia, we measured spirochete and platelet counts in mice infected with strains DAH p1 or p19 or with 5 other $B$ hermsii strains. Intraperitoneal inoculation into 129Sv/C57BL6 mice of $10^{6}$ of each these 7 strains resulted in peak spirochetemia by day 2 or 3 , but the bacterial concentrations at peak spirochetemia varied over a 50-fold range (Figure 6). The degree of thrombocytopenia correlated remarkably well with the degree of spirochetemia $(r=0.934$; Figure 6).

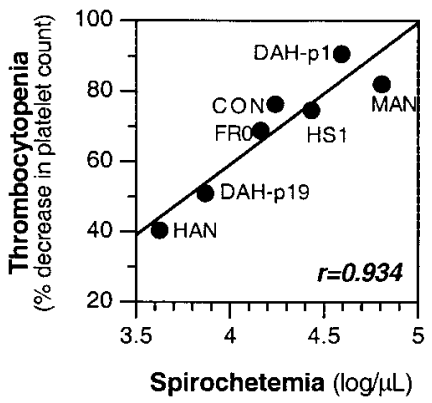

Figure 6. Thrombocytopenia is quantitatively correlated with spirochetemia $129 \mathrm{~Sv} / \mathrm{C} 57 \mathrm{BL} 6$ mice were inoculated intraperitoneally with $10^{6}$ spirochetes of the indicated $B$ hermsii strain. Spirochetemia, determined as described in "Materials and methods," peaked on day 2 or 3 after infection and is plotted on the x-axis. Platelet counts were also monitored, and the percentage of decrease from day 0 was calculated and plotted on the y-axis. The correlation coefficient $(r)$ is given. Sets of 2 mice were infected with each strain, and the results from each mouse were similar. For clarity, values from only 1 mouse are shown.

\section{Splenomegaly temporally correlates with thrombocytopenia during $B$ hermsii infection}

Accelerated clearance of platelets could result in enlargement of the major organs of the mononuclear phagocytic system, such as spleen and liver. To test this, 129Sv/C57BL6 mice were infected with $B$ hermsii DAH, and at 1- or 2-day intervals, mice were killed and spleen and liver weights and platelet and bacterial counts in the blood were determined. Liver weights were elevated by day 2 after infection and continued to increase through day 12, when the experiment was terminated (Figure 7Aiv). Strikingly, spleen weight correlated inversely with platelet count, peaking at 3 to 5 times the normal spleen weight on the days after thrombocytopenia (Figure 7Aii-iii). The temporal correlation between spirochetemia, thrombocytopenia, and splenomegaly was consistent with the hypothesis that platelets and bacteria are simultaneously cleared by the mononuclear phagocytic system.

Some forms of thrombocytopenia are associated with splenomegaly caused by splenic pooling of blood cells, ${ }^{46,47}$ raising the possibility that the thrombocytopenia of relapsing fever is a result of splenomegaly. To determine whether spleen is required for the induction of thrombocytopenia during relapsing fever, we monitored platelet counts in infected mice after splenectomy. As previously reported, ${ }^{25}$ mice that underwent splenectomy had more severe and prolonged spirochetemia than control mice (Figure 7Bi). These mice also had more severe and prolonged thrombocytopenia, suggesting that splenic clearance or pooling of platelets is not required for thrombocytopenia (Figure 7Bii). Nevertheless, the
A
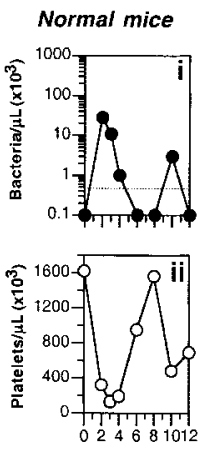

Days after infection
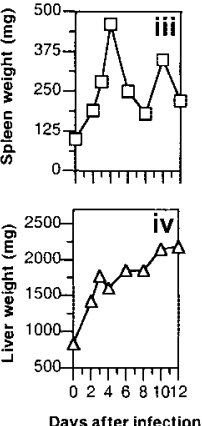

B
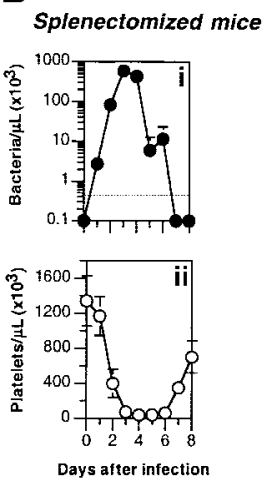

Figure 7. Splenomegaly temporally correlates with thrombocytopenia during B hermsii infection. (A) $129 \mathrm{~Sv} / \mathrm{C} 57 \mathrm{BL} 6$ mice were inoculated intraperitoneally with $10^{6} B$ hermsii DAH. On each of the indicated days after infection, 2 mice were killed and spirochete counts (i), platelet counts (ii), and spleen (iii) and liver (iv) weights were determined. (B) Spirochetemia (i) and thrombocytopenia (ii) in C57BL6 mice after splenectomy. 
occurrence of thrombocytopenia in mice after splenectomy does not preclude a role for the spleen in normal mice. It is possible that the liver, another organ of the mononuclear phagocytic system, is involved in platelet clearance during relapsing fever because this organ underwent significant enlargement during infection (Figure 7Aiv).

Circulating platelets bound to $B$ hermsii are not activated, and the activation-dependent integrin $\alpha_{\| 1} \beta_{3}$ is not required for the induction of thrombocytopenia

We previously showed that $B$ hermsii can activate human platelets in vitro, resulting in an enhanced bacterium-platelet attachment mediated by the activation-dependent integrin $\alpha_{\mathrm{IIb}} \beta_{3} .{ }^{12}$ Therefore, we examined the activation state of $B$ hermsii-bound platelets in infected murine blood. Spirochete-platelet complexes in freshly isolated blood from mice infected with strain DAH were identified by flow cytometry, and the activation state of bacterium-bound platelets was assessed by measuring the expression of P-selectin, a widely used platelet activation marker, ${ }^{21}$ and integrin $\alpha_{\mathrm{IIb}} \beta_{3}$, which is expressed at approximately 2-fold higher levels on activated rather than resting platelets. ${ }^{48} \mathrm{By}$ both measures, platelets bound to $B$ hermsii strain DAH displayed characteristics of resting platelets (Table 2). $B$ hermsii strain HS1 activates platelets in vitro more efficiently than does strain $\mathrm{DAH}$, raising the possibility that circulating platelets bound to this strain might demonstrate activated phenotype. ${ }^{12}$ Nevertheless, during murine infection, strain HS1 neither bound to platelets better than DAH nor induced higher levels of P-selectin or $\beta_{3}$-integrin (Table 2).

The observation that spirochete-bound circulating platelets in infected mice did not appear to be activated suggests that bacterial attachment to these circulating platelets is not mediated by integrin $\alpha_{\text {IIb }} \beta_{3}$. To address this issue, using a flow cytometric assay, we tested whether integrin $\alpha_{\mathrm{IIb}} \beta_{3}$ promoted bacterial attachment to purified murine platelets in vitro. As predicted, the activation of murine platelets resulted in increased bacterial binding (Figure 8A). This enhanced binding was likely caused by the activationdependent integrin $\alpha_{\mathrm{IIb}} \beta_{3}$ because it was abrogated by EDTA, which inhibits integrins, and it was not observed when bacterial binding was assessed using platelets from $\beta_{3}$-integrin-deficient mice. ${ }^{32}$ To test whether integrin $\alpha_{\text {II }} \beta_{3}$ contributed to bacterial binding in a measurable way in vivo, we infected $\beta_{3}$-integrindeficient 129Sv/C57BL6 mice with $B$ hermsii and measured bacterium-platelet complexes. The level of platelet binding by bacteria in the $\beta_{3}$-deficient mice was indistinguishable from that in wild-type 129Sv/C57BL6 controls, regardless of whether the infecting strain was $B$ hermsii DAH or HS1 (Figure 8B). Similarly, the presence or absence of functional $\alpha_{\mathrm{IIb}} \beta_{3}$ had no striking effect on the degree or kinetics of spirochetemia and thrombocytopenia

Table 2. Circulating platelets bound to $B$ hermsii are not activated

\begin{tabular}{lccc}
\hline & & \multicolumn{2}{c}{ Expression } \\
\cline { 3 - 4 } & Platelets bound, \% & P-selectin & 及3-integrin \\
\hline PMA, positive control & NA & 1.00 & 1.00 \\
No agonist, negative control & NA & 0.07 & 0.48 \\
DAH-bound platelets & 4.0 & 0.05 & 0.57 \\
HS1-bound platelets & 4.6 & 0.05 & 0.57 \\
\hline
\end{tabular}

Bacteria-bound platelets from the blood of mice infected with $B$ hermsii strain $\mathrm{DAH}$ or HS1 were quantitated and analyzed for the expression of P-selectin or $\beta_{3}$-integrin as described in "Materials and methods." $P$-selectin and $\beta_{3}$-integrin levels are expressed relative to the expression level of PMA-activated platelets, which was defined as 1.00. Shown are representative results of at least 2 independent experiments. NA indicates not applicable.
A
In vitro:

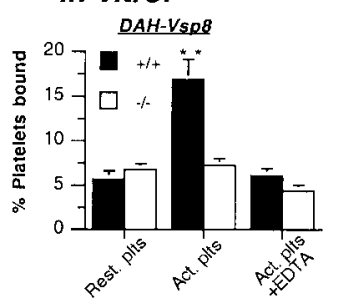

B

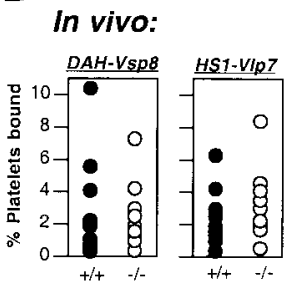

C
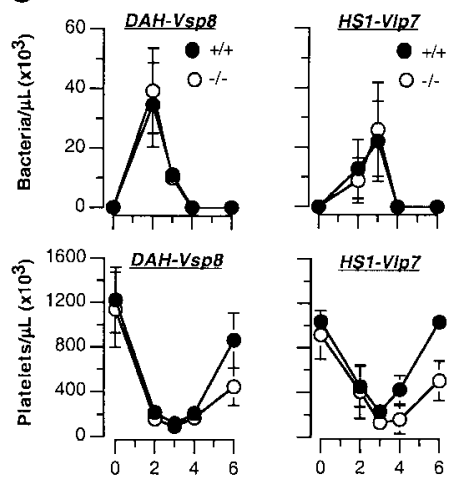

Days after infection
Figure 8. Activation-dependent integrin $\alpha_{\| b b} \beta_{3}$ is not required for bacteria attachment or induction of thrombocytopenia. (A) Resting (Rest) or thrombinactivated (Act.) wild-type $(+/+; \square)$ or $\beta_{3}$-integrin-deficient $(-/-; \square)$ mouse platelets were incubated with $B$ hermsii DAH-Vsp8 in the presence or absence of $10 \mathrm{mM}$ EDTA, and platelet binding was measured by flow cytometry. Shown are the means \pm SDs of 2 independent experiments. ${ }^{* *} P<.001$ with activated platelets from $-1-$ mice. (B-C) 129Sv/C57BL6 wild-type $(+/+; \bullet)$ or $\beta_{3}$-integrin-deficient $(-/-; \bigcirc)$ mice were inoculated intraperitoneally with $10^{6} B$ hermsii DAH or HS1. (B) At peak spirochetemia on day 2 after infection, the percentages of platelets with bound bacteria were quantified by flow cytometry (see "Materials and methods"). Shown are the means \pm SDs of 8 mice from each group. (C) On the indicated days afte infection, spirochete and platelet counts were determined. Shown are the means \pm SDs of 10 mice from each group.

induced by these 2 strains, though the return of platelet counts to near normal levels was marginally slower in $\beta_{3}$-deficient mice (Figure 8C). These results indicate that although integrin $\alpha_{\mathrm{IIb}} \beta_{3}$ is a critical receptor for high-level $B$ hermsii attachment to platelets in vitro, ${ }^{12}$ it does not significantly contribute to bacterial attachment to circulating platelets or to thrombocytopenia in the infected mouse.

\section{Discussion}

Thrombocytopenia is a prominent feature of human relapsing fever, but its etiology is unclear. In the current study, we have investigated the mechanism of relapsing fever-associated thrombocytopenia in a murine model. We established that $B$ hermsii infection of the mouse is indeed associated with severe thrombocytopenia and a functional defect in hemostasis. Megakaryopoiesis was stimulated, not reduced, during $B$ hermsii infection, suggesting that the thrombocytopenia was caused by accelerated platelet clearance rather than diminished production. Indeed we found that biotinylated platelets are cleared more rapidly in $B$ hermsiiinfected than in uninfected mice. Consistent with this hypothesis, the liver and spleen, organs involved in platelet clearance, were enlarged during infection. The 3 best-characterized causes of accelerated platelet clearance during infection-DIC, immune thrombocytopenic purpura, and splenic sequestration-did not appear to play a role. Fibrin degradation products characteristic of DIC were not detected, thrombocytopenia was induced in antibodydeficient (ie, SCID) mice, and splenectomy did not relieve $B$ hermsii-induced thrombocytopenia.

Having shown previously that $B$ hermsii binds to platelets in vitro, ${ }^{12}$ we tested whether such binding could also be observed 
during infection. Flow cytometric analysis of blood drawn from infected mice indicated that as many as $10 \%$ of circulating platelets were attached to spirochetes. It is likely that our flow cytometric quantitation of bacterial binding by circulating platelets at peak spirochetemia underestimated the true extent of bacterium-platelet interaction: spirochete-platelet complexes might have been rapidly cleared from the circulation and thereby rendered undetectable by flow cytometry. Thus, the $1 \%$ to $10 \%$ of circulating platelets that were found attached to spirochetes during peak spirochetemia may represent only a "snapshot" of the initial step in a dynamic process involving both the formation of spirochete-platelet complexes and their subsequent rapid clearance. Given that at peak spirochetemia the rate of $B$ hermsii replication ${ }^{12,25}$ is likely to be greater than the rate of platelet production, ${ }^{33}$ continuous formation and clearance of spirochete-platelet complexes could result in significant platelet loss. Consistent with this model, the degree of thrombocytopenia correlated well with the degree of spirochetemia; the timing of episodes of thrombocytopenia and splenomegaly were also strikingly correlated with episodes of spirochetemia.

Several features of the murine model for relapsing feverassociated thrombocytopenia parallel previous studies of relapsing fever in humans. For example, bone marrow biopsy samples from patients with thrombocytopenic relapsing fever revealed an increase in megakaryocyte numbers, ${ }^{17}$ and hepatosplenomegaly is a common feature of human infection. ${ }^{18,39,49,50}$ Patients with thrombocytopenic relapsing fever show no evidence of DIC, even in the presence of bleeding manifestations. ${ }^{17,18,49}$ Instead, plateletspirochete complexes have occasionally been observed in blood smears from patients with relapsing fever. ${ }^{17}$

In vitro $B$ hermsii activates human platelets and, concomitantly, the major platelet integrin $\alpha_{\mathrm{IIb}} \beta_{3}$ in a contact-dependent manner. ${ }^{12}$ This activation leads to high-level spirochete-platelet binding by way of $\alpha_{\mathrm{IIb}} \beta_{3}{ }^{12}$ In contrast, we obtained no evidence that circulating spirochete-bound platelets were activated during infection. These platelets did not differ from spirochete-free platelets in their expression of $\mathrm{P}$-selectin or $\beta_{3}$-integrin, 2 proteins that are expressed at higher levels on activated platelets than on resting platelets. In addition, the efficiency of bacterial binding to circulating platelets was similar in $\beta_{3}$-deficient and wild-type mice. It is possible that our inability to detect platelet activation represents the limitation of flow cytometry mentioned above-that is, only cells that remain in the circulation can be sampled and analyzed. It is also possible that $B$ hermsii, like several other platelet agonists such as epinephrine and serotonin, ${ }^{38}$ activates human platelets more efficiently than it does murine platelets.

Whether platelets undergo activation after spirochete binding during infection, the activation-dependent integrin $\alpha_{\mathrm{IIb}} \beta_{3}$ does not appear to be required for the induction of thrombocytopenia by $B$ hermsii. The kinetics and severity of thrombocytopenia were indistinguishable during infection of $\beta_{3}$-deficient and wild-type mice. The previously identified contact-dependent platelet activation activity of $B$ hermsii suggests that a binding pathway independent of $\alpha_{\mathrm{IIb}} \beta_{3}$ promotes an initial spirochete-platelet interaction. ${ }^{12}$ Presumably, if platelet clearance during infection is the result of bacterial attachment, this initial $\alpha_{\mathrm{IIb}} \beta_{3}$-independent attachment of spirochetes to resting platelets is sufficient to trigger this clearance, and the high-level binding mediated by $\alpha_{\mathrm{II}} \beta_{3}$ that we observed in vitro is apparently not required.

The precise outcome of platelet-bacterium attachment during infection is still unclear. One can speculate that by inducing thrombocytopenia and compromising hemostasis, this arthropodborne microbe expedites tick feeding and the subsequent transmission of the pathogen to the arthropod. The saliva of many blood-feeding arthropods, including Ornithodoros ticks that transmit relapsing fever spirochetes, contains inhibitors of platelet aggregation, ${ }^{51-53}$ presumably to facilitate feeding. It is notable that the agent of human granulocytic ehrlichiosis, which is transmitted by the same vector that transmits Borrelia burgdorferi, also induces thrombocytopenia in mice in a manner independent of an intact spleen or lymphocytes. ${ }^{54}$ Other arthropod-borne pathogens, such as Plasmodium and Trypanosoma, have been shown to bind platelets and induce thrombocytopenia. ${ }^{10,11}$ An alternative for spirocheteplatelet binding may be to promote bacterial attachment to the vessel wall, a step that might diminish clearance by the spleen and liver and lead to a population of immobilized spirochetes that, through replication, efficiently seeds the bloodstream with spirochetes. We previously showed that among a pair of $B$ turicatae strains likely to be isogenic, the ability to bind better to cultured endothelial cells was associated with the ability to achieve higher bacterial concentrations in the blood of infected animals. ${ }^{55}$ Finally, platelets have also been viewed as host defense cells that promote clearance of circulating microbes. Platelets can release bactericidal peptides, ${ }^{56}$ and, in several infection models, preexisting thrombocytopenia was associated with decreased microbial clearance. ${ }^{45,57-61}$ The ability to follow bacteremia, thrombocytopenia, bacteriumplatelet attachment, and tissue colonization in the murine model of relapsing fever described here will permit detailed analysis of the role of platelet-spirochete interactions during infection.

\section{Acknowledgments}

We thank Jorge Benach, James Coleman, and Joe Gebbia for generously providing training in animal infection techniques. We also thank Alan Barbour for providing $B$ hermsii HS1-Vlp7; Robert Woodland for providing CB.17/SCID mice; Jon Goguen, Jenifer Coburn, Guido Majno, Marc Barnard, and Larry Frelinger for providing stimulating discussions; and Yu Liu of the Diabetes and Endocrinology Research Center at University of Massachusetts Medical School for preparing bone marrow specimens.

\section{References}

1. Wilson JJ, Neame PB, Kelton JG. Infectioninduced thrombocytopenia. Semin Thromb Hemost. 1982;8:217-233

2. Gainor JP, Morton CA, Bell DR, Vincent PA, Minnear FL. Platelet phospholipids decrease vascular endothelial permeability via a novel signaling pathway independent of cAMP/protein kinase A. Ann N Y Acad Sci. 2000;905:315-318.

3. Axthelm MK, Krakowka S. Canine distemper virus-induced thrombocytopenia. Am J Vet Res. 1987;48:1269-1275.

4. Murphy MF, Metcalfe P, Waters AH, et al. Inci- dence and mechanism of neutropenia and thrombocytopenia in patients with human immunodeficiency virus infection. Br J Haematol. 1987;66: 337-340.

5. Neame PB, Kelton JG, Walker IR, Stewart IO, Nossel HL, Hirsh J. Thrombocytopenia in septicemia: the role of disseminated intravascular coagulation. Blood. 1980;56:88-92.

6. Edwards CN, Nicholson GD, Hassell TA, Everard $\mathrm{CO}$, Callender J. Thrombocytopenia in leptospirosis: the absence of evidence for disseminated intravascular coagulation. Am J Trop Med Hyg. 1986;35:352-354
7. Greenwood BM, Whittle HC. Coagulation studies in Gambian trypanosomiasis. Am J Trop Med Hyg. 1976;25:390-394.

8. Des Prez RM, Steckley S, Stroud RM, Hawiger J. Interaction of Histoplasma capsulatum with human platelets. J Infect Dis. 1980;142:32-39.

9. Skudowitz RB, Katz J, Lurie A, Levin J, Metz J. Mechanisms of thrombocytopenia in malignant tertian malaria. Br Med J. 1973;2:515-518.

10. Davis CE. Thrombocytopenia: a uniform complication of African trypanosomiasis. Acta Trop. 1982;39:123-133. 
11. Fajardo LF. Malarial parasites in mammalian platelets [letter]. Nature. 1973;243:298-299.

12. Alugupalli $K R$, Michelson $A D$, Barnard $M R$, et al. Platelet activation by a relapsing fever spirochete results in enhanced bacterium-platelet interaction via integrin $\alpha \mathrm{llb} \beta 3$ activation. Mol Microbiol. 2001; 39:330-340.

13. Southern PM, Sanford JP. Relapsing fever: clinical and microbiological review. Medicine. 1969;48:129-149.

14. Barbour AG. Linear DNA of Borrelia species and antigenic variation. Trends Microbiol. 1993;1:236239.

15. Stoenner HG, Dodd T, Larsen C. Antigenic variation of Borrelia hermsii. J Exp Med. 1982;156: 1297-1311.

16. Burgdorfer $\mathbf{W}$. The epidemiology of the relapsing fevers. In: Johnson RC, ed. The Biology of Parasitic Spirochetes. New York, NY: Academic Press; 1976:191-200.

17. Perine PL, Parry EHO, Vykovitch D, Warrell DA, Bryceson ADM. Bleeding in louse-borne relapsing fever, I: clinical studies with 37 patients. Trans R Soc Trop Med Hyg. 1971;65:776-781.

18. Salih SY, Mustafa D, Abdel Wahab SM, Ahmed MA, Omer A. Louse-borne relapsing fever, I: a clinical and laboratory study of 363 cases in the Sudan. Trans R Soc Trop Med Hyg. 1977; 71:43-48.

19. Butler T, Hazen P, Wallace CK, Awoke S, HabteMichael A. Infection with Borrelia recurrentis: pathogenesis of fever and petechiae. J Infect Dis. 1979;140:665-675.

20. Dworkin MS, Anderson DE Jr, Schwan TG, et al. Tick-borne relapsing fever in the northwestern United States and southwestern Canada. Clin Infect Dis. 1998;26:122-131.

21. Fox JE. Platelet activation: new aspects. Haemostasis. 1996;26(suppl 4):102-131.

22. Phillips DR, Charo IF, Scarborough RM. GPIIbIlla: the responsive integrin. Cell. 1991;65:359 362.

23. Newman K Jr, Johnson RC. T-cell-independent elimination of Borrelia turicatae. Infect Immun. 1984;45:572-576.

24. Connolly SE, Benach JL. Cutting edge: the spirochetemia of murine relapsing fever is cleared by complement-independent bactericidal antibodies. J Immunol. 2001;167:3029-3032.

25. Alugupalli KR, Gerstein RM, Chen J, SzomolanyiTsuda E, Woodland RT, Leong JM. The resolution of relapsing fever borreliosis requires $\lg \mathrm{M}$ and is concurrent with expansion of B1b lymphocytes. J Immunol. 2003;170:3819-3827.

26. Gebbia JA, Monco JC, Degen JL, Bugge TH, Benach JL. The plasminogen activation system enhances brain and heart invasion in murine relapsing fever borreliosis. J Clin Invest. 1999;103: 81-87.

27. Shamaei-Tousi A, Martin P, Bergh A, Burman N, Brannstrom T, Bergstrom S. Erythrocyte-aggregating relapsing fever spirochete Borrelia crocidurae induces formation of microemboli. J Infect Dis. 1999;180:1929-1938.

28. Alugupalli KR, Michelson AD, Barnard MR, Leong JM. Serial determinations of platelet counts in mice by flow cytometry. Thromb Haemost. 2001; 86:668-671.

29. Schwan TG, Hinnebusch BJ. Bloodstream-versus tick-associated variants of a relapsing fever bacterium. Science. 1998;280:1938-1940.

30. Thompson RS, Burgdorfer W, Russell R, Francis BJ. Outbreak of tick-borne relapsing fever in Spokane County, Washington. JAMA. 1969;210: 1045-1050.

31. Hinnebusch BJ, Barbour AG, Restrepo BI Schwan TG. Population structure of the relapsing fever spirochete Borrelia hermsii as indicated by polymorphism of two multigene families that encode immunogenic outer surface lipoproteins. Infect Immun. 1998:66:432-440.

32. Hodivala-Dilke KM, McHugh KP, Tsakiris DA, et al. $\beta 3$-Integrin-deficient mice are a model for Glanzmann thrombasthenia showing placental defects and reduced survival. J Clin Invest. 1999; 103:229-238.

33. Berger G, Hartwell DW, Wagner DD. P-Selectin and platelet clearance. Blood. 1998:92:44464452.

34. Manning KL, Novinger S, Sullivan PS, McDonald TP. Successful determination of platelet lifespan in $\mathrm{C} 3 \mathrm{H}$ mice by in vivo biotinylation. Lab Anim Sci. 1996;46:545-548.

35. Dejana E, Callioni A, Quintana A, de Gaetano G. Bleeding time in laboratory animals, II: a comparison of different assay conditions in rats. Thromb Res. 1979;15:191-197.

36. Burstein SA, Adamson JW, Erb SK, Harker LA Megakaryocytopoiesis in the mouse: response to varying platelet demand. J Cell Physiol. 1981; 109:333-341.

37. Schorer AE. Discordant effects on eicosanoids and fibrin degradation products in two murine models of antiphospholipid antibody. Thromb Res. 1997;85:295-304.

38. Tsakiris DA, Scudder L, Hodivala-Dilke K, Hynes RO, Coller BS. Hemostasis in the mouse (Mus musculus): a review. Thromb Haemost. 1999;81: 177-188.

39. Anda P, Sanchez-Yebra W, del Mar Vitutia M, et al. A new Borrelia species isolated from patients with relapsing fever in Spain. Lancet. 1996;348: 162-165.

40. George JN. Thrombocytopenia due to diminished or defective platelet production. In: Beutler E, Lichtman MA, Coller BS, Kipps TJ, eds. Williams Hematology. New York, NY: McGraw-Hill; 1995: 1281-1289.

41. Carr JM, McKinney M, McDonagh J. Diagnosis of disseminated intravascular coagulation: role of D-dimer. Am J Clin Pathol. 1989;91:280-287.

42. Gillis S, Eldor A. Immune thrombocytopenic purpura in adults: clinical aspects. Baillieres Clin Haematol. 1998;11:361-372.

43. Wadenvik $\mathrm{H}$, Stockelberg D, Hou M. Platelet proteins as autoantibody targets in idiopathic thrombocytopenic purpura. Acta Paediatr Suppl. 1998; 424:26-36.

44. Rand ML, Dean JA. Platelet function in autoimmune (idiopathic) thrombocytopenic purpura. Acta Paediatr Suppl. 1998;424:57-60.

45. Umekita LF, Carneiro SM, Mota I. One fate of epimastigote forms of Trypanosoma cruzi injected in mice: an ultrastructural study. J Parasitol. 1998; 84:1190-1195.

46. Aster RH. Pooling of platelets in the spleen: role in the pathogenesis of "hypersplenic" thrombocytopenia. J Clin Invest. 1966;45:645-657.

47. Pearson HA. The spleen and disturbances of splenic function. In: Nathan DG, Orkin SH, eds. Hematology of Infancy and Childhood. Vol 2. Philadelphia, PA: WB Saunders; 1987:10511068.

48. Woods VL, Wolff LE, Keller DM. Resting platelets contain a substantial centrally located pool of glycoprotein Ilb-IIla complex which may be accessible to some but not other extracellular proteins. J Biol Chem. 1986;261:15242-15251.

49. Ahmed MA, Abdel Wahab SM, Abdel Malik MO, et al. Louse-borne relapsing fever in the Sudan: a historical review and a clinico-pathological study. Trop Geogr Med. 1980;32:106-111.

50. Borgnolo G, Denku B, Chiabrera F, Hailu B. Louse-borne relapsing fever in Ethiopian children: a clinical study. Ann Trop Paediatr. 1993;13: 165-171.

51. Waxman L, Connolly TM. Isolation of an inhibito selective for collagen-stimulated platelet aggregation from the soft tick Ornithodoros moubata. J Biol Chem. 1993;268:5445-5449.

52. Karczewski J, Endris R, Connolly TM. Disagregin is a fibrinogen receptor antagonist lacking the Arg-Gly-Asp sequence from the tick, Ornithodoros moubata. J Biol Chem. 1994;269:6702-6708

53. Nienaber J, Gaspar AR, Neitz AW. Savignin, a potent thrombin inhibitor isolated from the salivary glands of the tick Ornithodoros savignyi (Acari: Argasidae). Exp Parasitol. 1999;93:82-91.

54. Borjesson DL, Simon SI, Tablin F, Barthold SW. Thrombocytopenia in a mouse model of human granulocytic ehrlichiosis. J Infect Dis. 2001;184 1475-1479.

55. Magoun L, Zuckert WR, Robbins D, et al. Variable small protein- (Vsp-) dependent and Vspindependent pathways for glycosaminoglycan recognition by relapsing fever spirochetes. Mol Microbiol. 2000;36:886-897.

56. Yeaman MR, Sullam PM, Dazin PF, Bayer AS Platelet microbicidal protein alone and in combination with antibiotics reduces Staphylococcus aureus adherence to platelets in vitro. Infect Immun. 1994;62:3416-3423.

57. Sullam PM, Frank U, Yeaman MR, Tauber MG, Bayer AS, Chambers HF. Effect of thrombocytopenia on the early course of streptococcal endocarditis. J Infect Dis. 1993;168:910-914.

58. Polack B, Delolme F, Peyron F. Protective role of platelets in chronic (Balb/C) and acute (CBA/J) Plasmodium berghei murine malaria. Haemostasis. 1998;27:278-285

59. Peyron F, Polack B, Lamotte D, Kolodie L, Ambroise-Thomas P. Plasmodium falciparum growth inhibition by human platelets in vitro. Parasitology. 1989;99(pt 3):317-322.

60. Umekita LF, Mota I. Role of platelets in the in vivo removal of Trypanosoma cruzi from circulation. Braz J Med Biol Res. 1990;23:593-596.

61. Umekita LF, Piazza RM, Mota I. Role of platelets and complement in the clearance of epimastigote forms of Trypanosoma cruzi. Braz J Med Biol Res. 1994;27:2391-2399. 